\title{
A check-list of parasites of percid fishes (Actinopterygii: Percidae) from the estuaries of the Polish coastal zone
}

\author{
J. MOROZINSKA-GOGOL
}

Department of Aquatic Ecology, Pomeranian Academy, ul. Arciszewskiego 22 b, 76-200 Słupsk, Poland, E-mail: jolamorozinska@poczta.onet.pl

\begin{abstract}
Summary
The present paper summarized data on parasites of percid fishes (Actinopterygii: Percidae), such as pike-perch, European perch and ruffe from estuaries of the Polish coastal zone are listed, based on published records and long-term surveys carried out by the author. Parasites are listed alphabetically, separately for each host and for each parasite group with notes on their location on the host and geographical distribution (with references). A total of 41 species (taxa) of parasites were recorded on pike-perch Sander lucioperca (6 Ciliophora, 1 Myxozoa, 1 Monogenea, 11 Digenea, 4 Cestoda, 6 Nematoda, 5 Acanthocephala, 1 Mollusca, 1 Hirudinea, 4 Copepoda and 1 Branchiura). European perch, Perca fluviatilis, harboured 60 parasites (6 Ciliophora, 4 Myxozoa, 1 Monogenea, 18 Digenea, 9 Cestoda, 10 Nematoda, 5 Acanthocephala, 1 Mollusca, 1 Hirudinea, 4 Copepoda and 1 Branchiura). Ruffe Gymnocephalus cernuus featured 43 parasites (1 Microsporidia, 7 Ciliophora, 3 Myxozoa, 1 Monogenea, 13 Digenea, 5 Cestoda, 8 Nematoda, 2 Acanthocephala, 1 Mollusca and 2 Copepoda).
\end{abstract}

Key words: parasites, Percidae, estuaries, Poland.

\section{Introduction}

There are two major estuaries at the Polish Baltic coast, namely the Oder River estuary emptying to the Pomeranian Bay and the Vistula River estuary associated with the Gulf of Gdańsk. The latter contains an inner shallow basin, known as - the Puck Bay. The Oder River estuary covers also the Szczecin Lagoon - and the Dąbie Lake. The major inland body of water within the Vistula River estuary in the Vistula Lagoon. A number of small estuaries, distributed along the Polish Baltic coast line include coastal lakes such as Koprowo, Liwia Łuża, Resko Przymorskie, Jamno, Bukowo, Kopań, Wicko, Gardno, Łebsko, Sarbsko, Żarnowieckie, and Drużno (Paturej, 2006). Two coastal lakes, Łebsko and Gardno, are situated within the Słowiński National Park (Trojanowski et al., 1991). Despite of similar origin, the estuaries on the Polish coast are characterised by different hydrological and hydrochemical conditions (Paturej, 2006; Trojanowski et al., 1991). All Polish estuaries are located as mixo-mesohaline waters (Paturej, 2006). The organisms, including fish and parasites, inhabiting those bodies of water, exhibit a broad tolerance to frequently changing conditions, especially salinity and temperature.

The aim of this study was to present the parasite fauna of Percidae from the Polish coastal zone and complete knowledge on the distribution of parasites affecting percids.

\section{Materials and Methods}

The present checklist of fish parasites was prepared based on the data published from 1933 to 2006 and covering the Polish coastal zone (with great emphasis on the Szczecin Lagoon, Vistula Lagoon, Lake Drużno, and Gulf of Gdańsk) and based on the present author's own survey of the coastal lakes (Middle Coast). In 1945 Polish borders (includig coastline) substantially changed. Length of the Polish coastline before 1945 was about $140 \mathrm{~km}$, whereas $770 \mathrm{~km}$ is now (Figure 1).

European perch and pike-perch, studied by the present author, were collected from 2001 to 2006, from the coastal lakes Bukowo, Kopań, and Łebsko (unpublished data marked as JM-G in the text). The fish were purchased from commercial fishermen and examined for ectoparasites and endoparasites under a dissecting microscope. The examination included the skin, fins, gills, eyes (lens and vitreous humour), body cavity, and visceral organs (stomach, intestine, liver and gonads). The collected parasites were subjected to standard parasitological procedures (Bylund et al., 1980). 
Identification of metazoan parasites was aided by taxonomic keys (Gusev, 1985; Bauer, 1987; Pojmańska, 1991; Niewiadomska, 2003; Grabda-Kazubska \& Okulewicz, 2005). Because of the high morphological similarity between metacercariae within the genus Diplostomum (cf. Niewiadomska, 1996, 2003), only the generic name is used. Apatemon annuligerum was regarded as a synonym of A. gracilis following the morphological and molecular evidence provided by Bell \& Sommerville (2002) and Bell et al. (2002). Others authors, cited in the present check-list, identified their parasites based on morphological characters, except Kempter et al. (2006), who examined parasites morphologically, biometrically, and genetically. Parasites recorded on pike-perch, European perch and ruffe are listed with notes about their location on the host and distribution with references.

\section{Results}

1. Parasites of pike-perch, (“zander"), Sander lucioperca (Linnaeus, 1758)

\section{Ciliophora}

Apiosoma campanulata (Timofeev, 1962); location: gills, skin; distribution: Szczecin Lagoon (Pilecka-Rapacz, 1980).

Capriniana piscium (Bütschli, 1858); ("Trichophrya piscium") location: gills, distribution: Szczecin Lagoon (Pilecka-Rapacz, 1980).

Epistylis lwoffi Fauré-Fremiet, 1943; location: gills, distribution: Szczecin Lagoon (Pilecka-Rapacz, 1980).

Trichodina domerguei Wallengreen, 1897; location: gills; distribution: Vistula Lagoon (Grabda, 1971), Lake Drużno (Kozicka, 1959).

Trichodina nigra Lom, 1960; location: gills, skin, nostrils; distribution: Szczecin Lagoon (Pilecka-Rapacz, 1980).

Trichodinella epizootica (Raabe, 1950); location: gills, skin, nostrils; distribution: Szczecin Lagoon (Pilecka-Rapacz, 1980), Lake Drużno (Kozicka, 1959).

\section{Myxozoa}

Henneguya psorospermica Thélohan, 1895; location: gills; distribution: Szczecin Lagoon (Pilecka-Rapacz, 1980).

\section{Monogenea}

Ancyrocephalus paradoxus Creplin, 1839; location: gills; distribution: Lake Jamno (Prost, 1966), Lake Lebsko (JMG), Gulf of Gdańsk (Rolbiecki \& Rokicki, 1996), Vistula Lagoon (Rolbiecki, 2003).

\section{Digenea}

Apophallus donicus (Skrjabin et Lindtrop, 1919); metacercaria; location: encysted in fins and skin; distribution: Szczecin Lagoon, Lake Dąbie (Wierzbicka \& Wierzbicki, 1973).

Azygia lucii (Müller, 1776); location: stomach; distribution: Gulf of Gdańsk (Rolbiecki \& Rokicki, 1996).

Brachyphallus crenatus (Rudolphi, 1802); location: sto- mach, intestine; distribution: Gulf of Gdańsk (Rokicki, 1975; Rolbiecki \& Rokicki, 1996), Vistula Lagoon (Rolbiecki, 2003).

Bucephalus polymorphus Baer, 1827; location: intestine, stomach; distribution: Lake Łebsko (JM-G), Gulf of Gdańsk (Rokicki, 1975; Rolbiecki \& Rokicki, 1996), Vistula Lagoon (Rolbiecki, 2003), Lake Drużno (Kozicka, 1959).

Bunodera luciopercae (Müller, 1776); location: intestine, pyloric caeca; distribution: Lake Łebsko (JM-G), Gulf of Gdańsk (Rokicki, 1975; Rolbiecki \& Rokicki, 1996), Vistula Lagoon (Rolbiecki, 2003), Lake Drużno (Kozicka, 1959).

Diplostomum spathaceum (Rudolphi, 1819); metacercaria; location: eye lens; distribution: Gulf of Gdańsk (Rolbiecki \& Rokicki, 1996), Lake Drużno (Kozicka, 1958).

Diplostomum spp.; metacercaria; location: eye lens; distribution: Lakes Kopań and Lebsko (JM-G), Gulf of Gdańsk (Rolbiecki et al., 1999), Vistula Lagoon ( Rolbiecki et al., 1999; Rolbiecki, 2003).

Ichthyocotylurus platycephalus (Creplin, 1825); metacercaria; location: intestine, heart wall, gills; distribution: Gulf of Gdańsk (Rolbiecki \& Rokicki, 1996; Rolbiecki et al., 1999), Vistula Lagoon (Rolbiecki et al., 1999; Rolbiecki, 2003;).

Rhipidocotyle illense (Ziegler, 1883); metacercaria and adult; location: metacercaria encysted in fins, adults intestine; distribution: Gulf of Gdańsk (Rokicki, 1975), Lake Drużno (Kozicka, 1958, 1959).

Rhipidocotyle campanula (Dujardin, 1845); location: intestine and pyloric caeca; distribution: Vistula Lagoon (Rolbiecki, 2003).

Tylodelphys clavata (von Nordmann, 1932); metacercaria; location: vitreous humour of eye; distribution: Lake Łebsko (JM-G), Gulf of Gdańsk (Rolbiecki \& Rokicki, 1996; Rolbiecki et al., 1999), Vistula Lagoon (Rolbiecki et al., 1999; Rolbiecki, 2003), Lake Drużno (Kozicka, 1958, 1959).

\section{Cestoda}

Bothriocephalus scorpii Müller, 1776; plerocercoid, adult; location: intestine and pyloric caeca; distribution: Gulf of Gdańsk (Rokicki, 1975; Rolbiecki \& Rokicki, 1996), Vistula Lagoon (Rolbiecki, 2003).

Caryophyllaeus laticeps (Pallas, 1781); location: intestine; distribution: Gulf of Gdańsk (Rokicki, 1975).

Eubothrium crassum (Bloch, 1779); plerocercoid; location: intestine; distribution: Vistula Lagoon (Rolbiecki, 2003).

Proteocephalus filicollis (Rudolphi, 1802); location: intestine and pyloric caeca; distribution: Vistula Lagoon (Rolbiecki, 2003).

\section{Nematoda}

Anguillicola crassus Kuwahara, Niimi et Itagaki, 1974; larvae; location: intestinal wall, liver, gonads; distribution: Vistula Lagoon (Rolbiecki, 2002, 2003).

Anisakis simplex (Rudolphi, 1809); larvae; location: body cavity; distribution: Vistula Lagoon (Rolbiecki\& Rokicki, 
2000; Rolbiecki, 2003).

Camallanus lacustris (Zoega, 1776); location: intestine; distribution: Lakes Kopań and Łebsko (JM-G), Gulf of Gdańsk (Rolbiecki \& Rokicki, 1996), Vistula Lagoon (Rolbiecki, 2003), Lake Drużno (Kozicka, 1959).

Camallanus truncatus (Rudolphi, 1814); location: intestine and pyloric caeca; distribution: Lakes Bukowo and Łebsko (JM-G), Gulf of Gdańsk (Rokicki, 1975; Rolbiecki \& Rokicki, 1996), Vistula Lagoon (Rolbiecki, 2003).

Cystidicoloides ephemeridarum (Linstow, 1872); location: intestine; distribution: Vistula Lagoon (Rolbiecki, 2003).

Hysterothylacium aduncum (Rudolphi, 1802); larvae and adult; location: intestine, intestinal wall, peritoneum, liver; distribution: Gulf of Gdańsk (Rolbiecki \& Rokicki, 1996), Vistula Lagoon (Rolbiecki, 2003).

\section{Acanthocephala}

Acanthocephalus lucii (Müller, 1776); location: intestine; distribution: Lake Łebsko (JM-G), Gulf of Gdańsk (Rokicki, 1975), Vistula Lagoon (Rolbiecki, 2003).

Corynosoma semerme (Forssel, 1904); cystacanth; location: intestinal wall; distribution: Gulf of Gdańsk (Rolbiecki \& Rokicki, 1996; Rolbiecki et al., 1999), Vistula Lagoon (Rolbiecki et al., 1999; Rolbiecki, 2003).

Corynosoma strumosum (Rudolphi, 1802); cystacanth; location: intestinal wall; distribution: Gulf of Gdańsk (Rolbiecki et al., 1999), Vistula Lagoon (Rolbiecki et al., 1999; Rolbiecki, 2003).

Neoechinorhynchus rutili (Müller, 1780); location: intestine; distribution: Gulf of Gdańsk (Rolbiecki \& Rokicki, 1996), Vistula Lagoon (Rolbiecki, 2003).

Pomphorhynchus laevis (Zoega, 1776); location: intestine; distribution: Gulf of Gdańsk (Rolbiecki \& Rokicki, 1996).

Mollusca: Bivalvia

Unionidae gen sp.; glochidia; location: fins; distribution: Vistula Lagoon (Rolbiecki, 2003).

\section{Hirudinea}

Piscicola geometra (Linnaeus, 1758); location: skin, mouth cavity, gills; distribution: Vistula Lagoon (Rolbiecki, 2003).

\section{Copepoda}

Achtheres sandrae Gadd, 1901; location: gills; distribution: ("A. percarum") Dąbie Lake and Szczecin Lagoon (Piasecki, 1993a), Lake Dąbie (Kempter et al., 2006; Piasecki et al., 2006); ("Achtheres percarum"); location: gills, mouth cavity; distribution: Odra River estuary (Piasecki, 1993b), Lakes Kopań and Łebsko (JM-G), Lake Wicko (Kozikowska, 1957), Gulf of Gdańsk (Rolbiecki \& Rokicki, 1996), Vistula Lagoon (Grabda, 1971; Rolbiecki, 2003).

Caligus lacustris Steenstrup et Lütken, 1861; chalimi and adults; location: chalimi on the fins, adults on the skin and in the mouth cavity; distribution: Lake Wicko (Kozikowska, 1957), Szczecin Lagoon (Piasecki, 1993a) Vistula Lagoon (Rolbiecki, 2003).
Ergasilus sieboldi von Nordmann, 1832; location: gills; distribution: Lakes Bukowo, Dąbie Lake and Szczecin Lagoon (Piasecki, 1993a), Kopań and Łebsko (JM-G), Gulf of Gdańsk (Rolbiecki \& Rokicki, 1996), Vistula Lagoon (Grabda, 1971; Rolbiecki, 2003), Lake Drużno (Kozicka, 1959).

\section{Branchiura}

Argulus foliaceus (Linnaeus, 1758); location: gills; distribution: Dąbie Lake and Szczecin Lagoon (Piasecki, 1993a), Lake Lebsko (JM-G), Gulf of Gdańsk (Rolbiecki \& Rokicki, 1996).

\section{Parasites of European perch, Perca fluviatilis Linnaeus, 1758}

\section{Ciliophora}

Apiosoma campalunata (Timofeev, 1962); location: gills, skin; distribution: Szczecin Lagoon (Pilecka-Rapacz, 1980).

Trichodina domerguei Wallengreen, 1897; location: gills; distribution: Vistula Lagoon (Grabda, 1971).

Trichodina nigra Lom, 1960; location: gills, skin, nostrils; distribution: Szczecin Lagoon (Pilecka-Rapacz, 1980).

Trichodina urinaria Dogiel, 1940; location: urinary tract; distribution: Szczecin Lagoon (Pilecka-Rapacz, 1980), Lake Resko (Sobecka \& Romanowski, 1999), Lake Drużno (Kozicka, 1959).

Trichodina sp.; location: gills; distribution: Lake Resko (Wierzbicka et al., 2005).

Trichodinella epizootica (Raabe, 1950); location: gills, skin, nostrils; distribution: Szczecin Lagoon (PileckaRapacz, 1980), Lake Resko (Wierzbicka et al., 2005).

\section{Myxozoa}

Henneguya creplini (Gurley, 1894); location: gills; distribution: Szczecin Lagoon (Romuk-Wodoracki, 1990).

Henneguya psorospermica Thélohan, 1895; location: gills; distribution: Szczecin Lagoon (Pilecka-Rapacz, 1980; Romuk-Wodoracki, 1990; Sobecka \& Romanowski, 1999), Vistula Lagoon (Grabda, 1971; Rolbiecki, 2003).

Henneguya wolinensis Romuk-Wodoracki, 1990; location: epidermis under scales; distribution: Szczecin Lagoon (Romuk-Wodoracki, 1990).

Myxobolus sp.; location: gills; distribution: Szczecin Lagoon (Sobecka \& Romanowski, 1999), Vistula Lagoon (Grabda, 1971), Lake Drużno (Kozicka, 1959).

\section{Monogenea}

Ancyrocephalus paradoxus Creplin, 1839; location: gills; distribution: Lake Drużno (Kozicka, 1959).

\section{Digenea}

Apophallus donicus (Skrjabin et Lindtrop, 1919); metacercaria; location: encysted in fins and skin; distribution: Szczecin Lagoon, Lakes Dąbie and Resko (Wierzbicka \& Wierzbicki, 1973).

Apatemon gracilis (Rudolphi, 1819) Szidat 1928; 
("Apatemon annuligerum"; "Tetracotyle annuligerum "); metacercaria; location: vitreous humour of eye; distribution: Lakes Bukowo, Kopań, and Łebsko (JM-G); ("Apatemon annuligerum"); location: vitreous humour of eye, brain, stomach; distribution: Lake Resko and adjacent area of the Baltic Sea (Wierzbicka et al., 2005), Gulf of Gdańsk (Rolbiecki et al., 1999), Vistula Lagoon (Rolbiecki et al., 1999; Rolbiecki, 2003); ("Tetracotyle annuligerum"); location: vitreous humour of eye, brain; distribution: Lake Drużno (Kozicka, 1958).

Azygia lucii (Müller, 1776); location: intestine, stomach, pharynx; distribution: Lake Resko (Wierzbicka et al., 2005), Lake Łebsko (JM-G), Gulf of Gdańsk (Rokicki, 1975), Lake Drużno (Kozicka, 1959).

Brachyphallus crenatus (Rudolphi, 1802); location: intestine; distribution: Lake Resko and adjacent area of the Baltic Sea (Wierzbicka et al., 2005).

Bucephalus polymorphus Baer, 1827; location: intestine; distribution: Lake Resko (Wierzbicka et al., 2005), Gulf of Gdańsk (Rokicki, 1975), Vistula Lagoon (Rolbiecki, 2003).

Bunodera luciopercae (Müller, 1776); location: intestine; distribution: Szczecin Lagoon (Sobecka \& Romanowski, 1999), Lake Resko and adjacent area of the Baltic Sea (Wierzbicka et al., 2005), Lake Łebsko (JM-G), Gulf of Gdańsk (Rokicki, 1975; Rolbiecki et al., 2002), Vistula River mouth (Rolbiecki et al., 2002), Vistula Lagoon (Rolbiecki, 2003), Lake Drużno (Kozicka, 1959).

Diplostomum spathaceum (Rudolphi, 1819); metacercaria; location: lens and vitreous humour of eye; distribution: Lake Drużno (Kozicka, 1958).

Diplostomum spp.; metacercaria; location: eye; distribution: Lake Resko and adjacent area of the Baltic Sea (Wierzbicka et al., 2005), Lakes Kopań and Łebsko (JM-G), Gulf of Gdańsk (Rolbiecki et al., 1999), Vistula River mouth (Rolbiecki et al., 2002), Vistula Lagoon (Rolbiecki et al., 1999; Rolbiecki, 2003).

Ichthyocotylurus platycephalus (Creplin, 1825); metacercaria; location: intestine; distribution: Lake Resko (Wierzbicka et al., 2005), Gulf of Gdańsk (Rolbiecki et al., 1999), Vistula Lagoon (Rolbiecki et al., 1999; Rolbiecki, 2003).

Ichthyocotylurus variegatus (Creplin, 1825); metacercaria; location: peritoneal cavity, peritoneum, intestine; distribution: Lake Resko and adjacent area of the Baltic Sea (Wierzbicka et al., 2005), Vistula Lagoon (Rolbiecki et al., 1999; Rolbiecki, 2003).

Nicolla skrjabini (Ivanitzky, 1928); location: intestine; distribution: Lake Resko (Wierzbicka et al., 2005), Gulf of Gdańsk (Rokicki, 1975).

Phyllodistomum pseudofolium Nybelin, 1926; location: urinary bladder, distribution: Lake Resko (Wierzbicka et al., 2005).

Posthodiplostomum brevicaudatum (von Nordmann, 1832); metacercaria; location: eye; distribution: Lake Łebsko (JM-G), Vistula Lagoon (Rolbiecki et al., 1999; Rolbiecki, 2003), Lake Drużno (Kozicka, 1958).

Posthodiplostomum cuticola (von Nordmann, 1832); metacercaria; location: stomach; distribution: Lake Resko and adjacent area of the Baltic Sea (Wierzbicka et al., 2005).

Rhipidocotyle illense (Ziegler, 1883); metacercaria and adult; location: metacercaria encysted in fins, intestine for adult; distribution: Gulf of Gdańsk (Rokicki, 1975), Lake Drużno (Kozicka, 1958, 1959).

Rhipidocotyle campanula (Dujardin, 1845); location: intestine; distribution: Lake Resko and adjacent area of the Baltic Sea (Wierzbicka et al., 2005).

Tylodelphys clavata (von Nordmann, 1932); metacercaria; location: vitreous humour of eye, brain; distribution: Szczecin Lagoon (Sobecka \& Romanowski, 1999), Lake Resko and adjacent area of the Baltic Sea (Wierzbicka et al., 2005), Lakes Bukowo, Kopań and Łebsko (JM-G), Gulf of Gdańsk (Rolbiecki et al., 1999, 2002), Vistula River mouth (Rolbiecki et al., 2002), Vistula Lagoon (Rolbiecki et al., 1999; Rolbiecki, 2003), Lake Drużno (Kozicka, 1958).

Tylodelphys podicipina Kozicka et Niewiadomska, 1960; metacercaria; location: vitreous humour of eye; distribution: Lake Drużno (Kozicka \& Niewiadomska, 1960).

\section{Cestoda}

Bothriocephalus scorpii (Müller, 1776); location: intestine, stomach; distribution: Lake Resko and adjacent area of the Baltic Sea (Wierzbicka et al., 2005), Lake Łebsko (JM-G), Gulf of Gdańsk (Rokicki, 1975).

Diphyllobothrium latum (Linnaeus, 1758); plerocercoid; location: liver; distribution: Szczecin Lagoon (Sobecka \& Romanowski, 1999), Lake Drużno (Kozicka, 1959).

Eubothrium crassum (Bloch, 1779); plerocercoid; location: intestine; distribution: Szczecin Lagoon (Sobecka \& Romanowski, 1999), Vistula River mouth (Rolbiecki et al., 2002), Vistula Lagoon (Rolbiecki, 2003).

Ligula sp.; plerocercoid; location: intestine, stomach; distribution: the Baltic Sea near Dźwirzyno (Wierzbicka et al., 2005).

Proteocephalus filicollis (Rudolphi, 1802); location: intestine and pyloric caeca; distribution: Lake Łebsko (JMG), Vistula Lagoon (Rolbiecki, 2003).

Proteocephalus percae (Müller, 1780); location: intestine; distribution: Szczecin Lagoon (Sobecka \& Romanowski, 1999), Lake Resko and adjacent area of the Baltic Sea (Wierzbicka et al., 2005), Lake Łebsko (JM-G), Baltic Sea near Hel (Markowski, 1933), Gulf of Gdańsk (Rokicki, 1975), Vistula River mouth (Rolbiecki et al., 2002), Vistula Lagoon (Rolbiecki, 2003), Lake Drużno (Kozicka, 1959).

Proteocephalus sp.; location: intestine; distribution: Lake Drużno (Kozicka, 1959).

Triaenophorus crassus Forel, 1868; plerocercoid; location: encysted in liver; distribution: Gulf of Gdańsk (Rokicki, 1975).

Triaenophorus nodulosus (Pallas, 1781); plerocercoid and adult; location: liver, body cavity for plerocercoid, intestine for adult; distribution: Szczecin Lagoon (Sobecka 
\& Romanowski, 1999), Lake Resko and adjacent area of the Baltic Sea (Wierzbicka et al., 2005), Lakes Bukowo and Łebsko (JM-G), Baltic Sea near Hel (Markowski, 1933), Gulf of Gdańsk (Rokicki, 1975; Rolbiecki et al., 2002), Vistula River mouth (Rolbiecki et al., 2002), Vistula Lagoon (Rolbiecki, 2003).

\section{Nematoda}

Anguillicola crassus Kuwahara, Niimi et Itagaki, 1974; larvae; location: intestinal wall, liver, gonads; distribution: Vistula Lagoon (Rolbiecki, 2002, 2003).

Anisakis simplex (Rudolphi, 1809); larvae; location: body cavity; distribution: Vistula Lagoon (Rolbiecki, 2003).

Camallanus lacustris (Zoega, 1776); location: intestine and pyloric caeca; distribution: Lake Resko and adjacent area of the Baltic Sea (Wierzbicka et al., 2005), Lake Gardno (Grabda, 1971), Lake Łebsko (JM-G), Gulf of Gdańsk (Rokicki, 1975), Vistula River mouth (Rolbiecki et al., 2002), Vistula Lagoon (Rolbiecki, 2003), Lake Drużno (Kozicka, 1959).

Camallanus truncatus (Rudolphi, 1814); location: intestine and pyloric caeca; distribution: Lakes Kopań and Łebsko (JM-G), Gulf of Gdańsk, Vistula River mouth (Rolbiecki et al., 2002), Vistula Lagoon (Rolbiecki, 2003).

Cucullanus sp.; location: intestine; distribution: Lake Resko and adjacent area of the Baltic Sea (Wierzbicka et al., 2005).

Cystidicoloides ephemeridarum (Linstow, 1872); location: intestine; distribution: Vistula Lagoon (Rolbiecki, 2003).

Desmidocercella numidica (Seurat, 1920); larvae; location: vitreous humour of eye; distribution: Lake Drużno (Kozicka, 1959).

Eustrongylides excisus Jägerskiöld, 1909; larvae; location: encysted in the intestine; distribution: Lake Resko and adjacent area of the Baltic Sea (Wierzbicka et al., 2005).

Hysterothylacium aduncum (Rudolphi, 1802); larvae and adult; location: intestine, intestinal wall, peritoneum, liver; distribution: Szczecin Lagoon (Sobecka \& Romanowski, 1999), Lake Resko and adjacent area of the Baltic Sea (Wierzbicka et al., 2005), Lakes Bukowo and Łebsko (JMG), Gulf of Gdańsk (Rolbiecki et al., 2002), Vistula River mouth (Rolbiecki et al., 2002), Vistula Lagoon (Rolbiecki, 2003); ("Contracaecum aduncum"); location: intestine; distribution: Baltic Sea near Hel (Markowski, 1933), Gulf of Gdańsk (Rokicki, 1975).

Raphidascaris acus (Bloch, 1779); location: intestine; distribution: Lake Łebsko (JM-G), Vistula Lagoon (Rolbiecki, 2003).

\section{Acanthocephala}

Acanthocephalus clavula (Dujardin 1845); location: intestine; distribution: Lake Kopań (JM-G), Gulf of Gdańsk (Rolbiecki et al., 2002),

Acanthocephalus lucii (Müller, 1776); location: intestine and pyloric caeca; distribution: Lake Resko and adjacent area of the Baltic Sea (Wierzbicka et al., 2005), Lake Łebsko (JM-G), Gulf of Gdańsk (Rokicki, 1975; Rolbiecki et al., 2002), Vistula River mouth (Rolbiecki et al., 2002),
Vistula Lagoon (Rolbiecki, 2003), Lake Drużno (Styczyńska, 1958; Wiśniewski, 1958; Kozicka, 1959).

Echinorhynchus gadi (Müller, 1776); location: intestine; distribution: Szczecin Lagoon (Sobecka \& Romanowski, 1999), Lake Resko and adjacent area of the Baltic Sea (Wierzbicka et al., 2005).

Neoechinorhynchus rutili (Müller, 1780); location: intestine; distribution: Lake Resko and adjacent area of the Baltic Sea (Wierzbicka et al., 2005), Lake Lebsko (JM-G), Gulf of Gdańsk (Rokicki, 1975).

Pomphorhynchus laevis (Zoega, 1776); location: intestine; distribution: Lake Łebsko (JM-G).

\section{Mollusca: Bivalvia}

Unionidae gen sp.; glochidia; location: gills, fins; distribution: Szczecin Lagoon (Sobecka \& Romanowski, 1999), Lake Resko and adjacent area of the Baltic Sea (Wierzbicka et al., 2005), Vistula Lagoon (Rolbiecki, 2003).

\section{Hirudinea}

Piscicola geometra (Linnaeus, 1758); location: operculum, gills, skin, mouth cavity; distribution: Szczecin Lagoon (Sobecka \& Romanowski, 1999), Lake Łebsko (JM-G), Vistula Lagoon (Grabda, 1971; Rolbiecki, 2003).

\section{Copepoda}

Achtheres percarum von Nordmann, 1832; location: gills, buccal cavity; distribution: Szczecin Lagoon (Kozikowska, 1957), Lake Dąbie (Kempter et al., 2006; Piasecki et al., 2006; Piasecki \& Kuźmińska, 2007), Lake Resko and adjacent area of the Baltic Sea (Wierzbicka et al., 2005), Lake Kopań (JM-G), Mouth of Vistula River (Grabda, 1967; Rolbiecki et al., 2002), Vistula Lagoon (Grabda, 1971), Lake Drużno (Kozicka, 1959).

Caligus lacustris Steenstrup et Lütken, 1861; chalimi and adults; location: chalimi on the fins, adults on the skin and in the mouth cavity; distribution: Vistula Lagoon (Rolbiecki, 2003).

Ergasilus sieboldi von Nordmann, 1832; location: gills; distribution: Lake Wicko (Kozikowska, 1957), Lakes Kopań and Łebsko (JM-G), Vistula Lagoon (Grabda, 1971; Rolbiecki, 2003), Lake Drużno (Kozicka, 1958, 1959).

Ergasilus sp.; location: gills; distribution: Lake Resko and adjacent area of the Baltic Sea (Wierzbicka et al., 2005).

\section{Branchiura}

Argulus foliaceus (Linnaeus, 1758); location: skin, gills, mouth; distribution: Lake Resko (Wierzbicka et al., 2005), Lake Wicko (Kozikowska, 1957), Vistula Lagoon (Grabda, 1971), Lake Drużno (Kozicka, 1959).

3. Parasites of ruffe, Gymnocephalus cernuus (Linnaeus, 1758)

Microsporidia

Glugea acerinae Jirovec, 1930; location: whitish, intestinal 
wall; distribution: Szczecin Lagoon (Wierzbicka et al., 1982), Vistula Lagoon (Rolbiecki, 2003).

\section{Myxozoa}

Henneguya creplini (Gurley, 1894); location: gills; distribution: Vistula Lagoon (Grabda, 1971).

Myxobolus magnus Awerinzew, 1913; location: vitreous humour of eye, iris; distribution: Szczecin Lagoon (Wierzbicka et al., 1982).

Myxobolus muelleri Bütschli, 1882; location: kidney; distribution: Szczecin Lagoon (Wierzbicka et al., 1982).

\section{Ciliophora}

Apiosoma campalunata (Timofeev, 1962); location: gills, skin; distribution: Szczecin Lagoon (Pilecka-Rapacz, 1980).

Capriniana piscium Bütschli, 1858; ("Trichophrya piscium") location: gills, distribution: Szczecin Lagoon (Pilecka-Rapacz, 1980).

Epistylis lwoffi Fauré-Fremiet, 1943; location: gills, distribution: Szczecin Lagoon (Pilecka-Rapacz, 1980).

Trichodina domerguei Wallengreen, 1897; location: gills; distribution: Vistula Lagoon (Grabda, 1971).

Trichodina domerguei f. esocis Lom, 1960; location: gills, skin; distribution: Szczecin Lagoon (Pilecka-Rapacz, 1980).

Trichodina nigra Lom, 1960; location: gills, skin, nostrils; distribution: Szczecin Lagoon (Pilecka-Rapacz, 1980).

Trichodinella epizootica (Raabe, 1950); location: gills, skin, nostrils; distribution: Szczecin Lagoon (PileckaRapacz, 1980; Wierzbicka et al., 1982).

\section{Monogenea}

Dactylogyrus amphibothrium Wagener, 1857; location: gills; distribution: Szczecin Lagoon (Wierzbicka et al., 1982), Vistula Lagoon (Rolbiecki, 2003).

\section{Digenea}

Apophallus donicus (Skrjabin et Lindtrop, 1919); metacercaria; location: encysted in the caudal fin and on the dorsal side skin; distribution: Szczecin Lagoon (Wierzbicka \& Wierzbicki, 1973; Wierzbicka et al., 1982), Lake Dąbie (Wierzbicka \& Wierzbicki, 1973).

Bucephalus polymorphus Baer, 1827; metacercaria and adult; location: metacercaria encysted in muscles close to the caudal fin and in the fin, gills and around the urinary bladder, adult in intestine; distribution: Szczecin Lagoon (Wierzbicka et al., 1982).

Bunodera luciopercae (Müller, 1776); location: intestine; distribution: Szczecin Lagoon (Wierzbicka et al., 1982), Gulf of Gdańsk (Rokicki, 1975), Lake Drużno (Kozicka, 1959).

Crepidostomum farionis (Müller, 1784); location: intestine; distribution: Szczecin Lagoon (Wierzbicka et al., 1982).

Diplostomum spathaceum (Rudolphi, 1819); metacercaria; location: lens and sometimes vitreous humour of eye; distribution: Lake Drużno (Kozicka, 1958).
Diplostomum spp.; metacercaria; location: eye lens; distribution: Szczecin Lagoon (Wierzbicka et al., 1982), Vistula Lagoon (Rolbiecki et al., 1999; Rolbiecki, 2003). Ichthyocotylurus platycephalus (Creplin, 1825); metacercaria; location: various internal organs, most often in the heart; distribution: Szczecin Lagoon (Wierzbicka et al., 1982), Vistula Lagoon (Rolbiecki et al., 1999; Rolbiecki, 2003).

Ichthyocotylurus variegatus (Creplin, 1825); metacercaria; location: various internal organs, most often in the swim bladder and peritoneal membrane, also the urinary bladder, liver, intestine, around the heart; distribution: Szczecin Lagoon (Wierzbicka et al., 1982), Vistula Lagoon (Rolbiecki et al., 1999; Rolbiecki, 2003).

Nicolla skrjabini (Ivanitzky, 1928); location: intestine; distribution: Szczecin Lagoon (Wierzbicka et al., 1982), Gulf of Gdańsk (Rokicki, 1975).

Phyllodistomum folium (Olfers, 1816); location: urinary bladder; distribution: Szczecin Lagoon (Wierzbicka et al., 1982).

Phyllodistomum pseudofolium Nybelin, 1926; location: urinary bladder; distribution: Szczecin Lagoon (Wierzbicka et al., 1982).

Rhipidocotyle illense (Ziegler, 1883); metacercaria and adult; location: metacercaria encysted in fins, adult in intestine; distribution: Lake Drużno (Kozicka, 1958, 1959). Tylodelphys clavata (von Nordmann, 1932); metacercaria; location: vitreous humour of eye; distribution: Szczecin Lagoon (Wierzbicka et al., 1982), Vistula Lagoon (Rolbiecki et al., 1999; Rolbiecki, 2003), Lake Drużno (Kozicka, 1958).

Cestoda

Bothriocephalus scorpii (Müller, 1776); location: intestine, stomach; distribution: Gulf of Gdańsk (Rokicki, 1975).

Caryophyllaeus laticeps (Pallas, 1781); location: intestine; distribution: Szczecin Lagoon (Wierzbicka et al., 1982), Gulf of Gdańsk (Rokicki, 1975).

Eubothrium crassum (Bloch, 1779); plerocercoid; location: intestine; distribution: Vistula Lagoon (Rolbiecki, 2003).

Proteocephalus filicollis (Rudolphi, 1802); location: intestine and pyloric caeca; distribution: Vistula Lagoon (Rolbiecki, 2003).

Proteocephalus sp.; location: intestine; Szczecin Lagoon (Wierzbicka et al., 1982).

\section{Nematoda}

Anguillicola crassus Kuwahara, Niimi et Itagaki, 1974; larvae; location: intestinal wall, liver, gonads; distribution: Vistula Lagoon (Rolbiecki, 2002, 2003).

Camallanus lacustris (Zoega, 1776); location: intestine; distribution: Lake Drużno (Kozicka, 1959).

Camallanus truncatus (Rudolphi, 1814); location: intestine; distribution: Gulf of Gdańsk (Rokicki, 1975).

Eustrongylides mergorum (Rudolphi, 1809); larvae; location: encysted on the intestine; distribution: Vistula Lagoon (Rolbiecki et al., 1999; Rolbiecki, 2003).

Eustrongylides sp.; larvae; location: encysted on the 
external intestinal wall; distribution: Szczecin Lagoon (Wierzbicka et al., 1982).

Hysterothylacium aduncum (Rudolphi, 1802); larvae; location: intestinal wall, peritoneum, liver; distribution: Vistula Lagoon (Rolbiecki, 2003).

Schulmanella petruschewskii (Schulman, 1948); ("Capillaria petruschewskii") location: liver; distribution: Szczecin Lagoon (Wierzbicka et al., 1982); Vistula Lagoon (Rolbiecki, 2003).

\section{Acanthocephala}

Acanthocephalus anguillae (Müller, 1780); location: intestine; distribution: Szczecin Lagoon (Wierzbicka et al., 1982).

Acanthocephalus lucii (Müller, 1776); location: intestine; distribution: Szczecin Lagoon (Wierzbicka et al., 1982), Gulf of Gdańsk (Rokicki, 1975), Lake Drużno (Styczyńska, 1958; Wiśniewski, 1958; Kozicka, 1959).

Mollusca: Bivalvia

Unionidae gen sp.; glochidia; location: gills, fins; distribution: Vistula Lagoon (Rolbiecki, 2003).

\section{Copepoda}

Caligus lacustris Steenstrup et Lütken, 1861; location: skin, mouth cavity; distribution: Vistula Lagoon (Rolbiecki, 2003).

Ergasilus sieboldi von Nordmann, 1832; location: gills; distribution: Szczecin Lagoon (Kozikowska, 1957; Wierzbicka et al., 1982), Vistula Lagoon (Grabda, 1971; Rolbiecki, 2003).

\section{Discussion}

The parasites found represented 60 species (taxa) of parasites on the European perch, 43 on the ruffe and 41 on the pike-perch. Percid fishes were noted as new intermediate host for Anguillicola crassus (Rolbiecki, 2002, 2003). Pike-perch and European perch, like the three-spined stickleback, were also proved to be a paratenic host for Anisakis simplex in the coastal waters (Rolbiecki \& Rokicki, 2000; Rolbiecki, 2003; MorozińskaGogol, 2006). Pike-perch and ruffe were shown to be a postcyclic hosts of caryophyllidean tapeworm Caryophyllaeus laticeps.

The parasite fauna of percid fishes consist of allogenic parasites (Apatemon gracilis, Diplostomum spathaceum, Diplostomum spp., Ichthyocotylurus platycephalus, Ichthyocotylurus variegatus, Posthodiplostomum brevicaudatum, Posthodiplostomum cuticola, Tylodelphys clavata, Tylodelphys podicipina, Diphyllobothrium latum, Ligula sp., Anisakis simplex, Desmidocercella numidica, Eustrongylides excisus, Eustrongylides mergorum, Eustrongylides sp., Corynosoma semerme, Corynosoma strumosum) and autogenic group (the remaining parasites). The parasites collected were predominantly freshwater species. They have been known from percid fishes inhabiting freshwater habitats (e.g. Wierzbicki, 1970;
Waluga \& Własow, 1988) and also from other freshwater fish species from the coastal water of the Baltic Sea. Because of low salinity, marine parasites were not very frequent in the estuaries covered by the present paper and occurred in fish from the water bodies affected by brackish waters of the Baltic Sea. Only Bothriocephalus scorpii, Brachyphallus crenatus, Anisakis simplex, Hysterothylacium aduncum, and Echinorhynchus gadi are typical marine species (Wierzbicka et al., 2005; Zander \& Reimer, 2002).

\section{References}

BAUER, O. N. (1987): Guide of parasites in freshwater fishes of the USRR Fauna. T. III. Nauka, Leningrad

Bell, A. S., Sommerville, C. (2002): Molecular evidence for the synonymy of two species of Apatemon Szidat, 1928, A. gracilis (Rudolphi, 1819) and $A$. annuligerum (von Nordmann, 1832) (Digenea: Strigeidae) parasitic as metacercariae in British fishes. J. Helminthol. 76: $193-198$

Bell, A. S, Sommerville, C., Gibson, D. I. (2002): Multivariate analyses of morphometrical features from Apatemon gracilis (Rudolphi, 1819) Szidat, 1928 and A. annuligerum (v. Nordmann, 1832) (Digenea: Strigeidae) metacercariae. Syst. Parasitol. 51: 121 - 133

Bylund, G., Fagerholm, H.-P., CAlenius, G., Wikgren, B.-J., WikströM, M. (1980): Parasites of Finland. II. Methods for studying parasite fauna in fish. Acta Akad. Aboen. Ser. B 40: 1 - 23

GrabdA, J. (1967): Parasitic Copepoda and Branchiura. Catalogue of fauna of Poland. Part XII, z. 5. PWN, Warszawa

GrabDA, J. (1971): Catalogue of parasitic fauna of Poland. Part II. Parasites of cyclostomes and fishes. PWN, Warszawa

GrabDA-KaZubSKA, B., OKulewiCZ, A. (2005): Guide of parasites in fishes of Poland - Nematoda. Polskie Towarzystwo Parazytologiczne, Warszawa

GuSEV, A. V. (1985): Guide of parasites in freshwater fishes of the USRR Fauna. T. II. Nauka, Leningrad

Kempter, J., PiAsECKI, W., WiĘSKI, K., KRAWCZYK, B. (2006): Systematic position of copepods of the genus Achtheres (Crustacea: Copepoda: Siphonostomatoida) parasitizing perch, Perca fluviatilis L., and zander, Sander lucioperca (L.). J. Fish Dis. 29: 103 - 113

KozicKA, J. (1958): Diseases of fishes of Drużno Lake. [Parasitofauna of the biocenosis of Drużno Lake - part VII]. Acta Parasitol. Pol. 6: 393 - 432

KozicKA, J. (1959): Parasites of fishes of Drużno Lake. [Parasitofauna of the biocenosis of Drużno Lake - part VIII]. Acta Parasitol. Pol. 7: $1-72$

Kozicka, J., Niewiadomska, K. (1960): Studies on the biology and taxonomy of trematodes of the genus Tylodelphys Diesing, 1850 (Diplostomatidae). Acta Parasitol.Pol. 8: $379-401$

KozIKowsKA, Z. (1957): Parasitic crustaceans (Crustacea parasitica) of Poland, part I. Parasites from the mouth of 
the Odra River. Zool. Pol. 8: $217-270$

MARKOWSKI, S. (1933): Die Eingeweidewurmer der Fische des polonischen Balticums (Trematoda, Cestoda, Nematoda, Acanthocephala). Arch. Hydrobiol. Ryb., Suwatki 7: $1-58$

MOROZIŃSKA-GOGOL, J. (2006): A checklist of parasites recorded on sticklebacks (Actinopterygii: Gasterosteidae) from Poland. Parasitol. Int. 55: $69-73$

NiEWIAdOMSKA, K. (1996): The genus Diplostomum taxonomy, morphology and biology. Acta Parasitol. 41: 55 $-66$

NIEWIADOMSKA, K. (2003): Guide of Parasites in fishes of Poland - Digenea. Polskie Towarzystwo Parazytologiczne, Warszawa

PAturej, E. (2006): Estuaries of the Polish Baltic Coastal Zone. Baltic Coast. Zone 10: 83 - 96

PIASECKI, W. (1993a): Crustacean parasites from three fish species from the Odra River estuary, Poland. Acta Ichthyol. Piscat. 23: $77-87$

PIASECKI, W. (1993b): Interantennary organ of Achtheres percarum von Nordmann, 1832 (Copepoda, Siphonostomatoida, Lernaepodidae) and some other new details of its external morphology. Acta Parasitol. 38: $36-$ 40

PiAsecKi, W., KuŹMIŃSKA, E. (2007): Developmental stages of Achtheres percarum (Crustacea: Copepoda), parasitic on European perch, Perca fluviatilis (Actinopterygii: Perciformes). Acta Ichthyol. Piscat. 37: 117-128

Piasecki, W., Sękowska-Jakubowska, A., Sobecka, E. (2006): Comparative description of males of two species of Achtheres von Nordmann, 1832 (Copepoda: Siphonostomatoida: Lernaepodidae) infecting zander and European perch. Folia Parasitol. 53: 211 - 216

PILECKA-RAPACZ, M. (1980): Protozoan parasites of the Szczecin Firth Percidae. Zesz. Nauk. Akad. Rol., Szczecin, ser. Ryb. Mor. 82: $75-86$

POJMAŃSKA, T. (1991): Guide of parasites in fishes of Poland - Cestoda. Polskie Towarzystwo Parazytologiczne, Warszawa

Prost, M. (1966): Monogenoidea. Catalogue of fauna of Poland. Part IV, z. 1. PWN, Warszawa

RoKICKI, J. (1975): Helminth fauna of fishes of the Gdańsk Bay (Baltic Sea). Acta Parasitol. Pol. 23: 37 - 84

ROLBIECKI, L. (2002): On the role of paratenic hosts in the life cycle of the nematode Anguillicola crassus in the Vistula Lagoon, Poland. Acta Ichthyol. Piscat. 32: 109-116 ROLBIECKI, L. (2003): Diversity of the parasite fauna of cyprinid (Cyprinidae) and percid (Percidae) fishes in the Vistula Lagoon, Poland. Wiad. parazytol. 49: 125 - 164 ROLBIECKI L., ROKICKI J. (1996): Parasitic metazoa of zander (Stizostedion lucioperca L.) in the Gulf of Gdańsk. Crangon 1: $73-85$
ROLBIECKI, L., ROKICKI, J. (2000): The occurrence of the nematodes Anisakis simplex pathogenic to man in pikeperch from the Vistula Lagoon, Poland. Wiad. parazytol. 46: $397-402$

RolBIECKI, L., ROKICKI, J., SzUGAJ, K. (2002): Variability of perch, Perca fluviatilis L., helminth fauna in the Gulf of Gdańsk, Baltic Sea. Oceanol. Stud. 31: 43 - 50

ROlBIECKI, L., ROKICKI, J., MOROZIŃSKA-GOGOL, J., CHIBANI, M. (1999): Larval stages of helminths in fish from the Vitula Lagoon and the Gulf of Gdańsk in relation to bird occurrence. Bull. Sea Fish. Inst. 2: $51-60$

ROMUK-WODORACKI, D. (1990): Henneguya wolinensis n.sp. (Myxosporea) from perch Perca fluviatilis L. from Szczecin Firth, Poland. Acta Ichthyol. Piscat. 20: 91 - 96

SoBecKA, E., RomAnOwsKi, P. (1999): Parasites of perch Perca fluviatilis L. from the Szczecin Lagoon. 5th Int Symp fish parasit. 9-13 August, 1999, Česke Budejovice, Czech Republic, 136

STYCZYŃSKA, E. (1958): Acanthocephala of the biocoenosis of Drużno Lake (Parasitolofauna of the biocoenosis of Drużno Lake - part VI). Acta Parasitol. Pol. 6: $195-211$

Trojanowski, J., Trojanowska, C., KorzenIEwsKi, K. (1991): Trophic state of coastal lakes. Pol. Archiw. Hydrobiol. 38: 23 - 34

Waluga, D., WŁAsow, T. (1988): The occurrence of parasites in bream (Abramis brama L.), roach (Rutilus rutilus L.) and pike perch (Stizostedion lucioperca L.) in the Włocław Dam Reservoir on the river Vistula. Wiad. parazytol. 34: $65-75$

WIERZBICKA, J., WIERZBICKI, K. (1973): Metacercariae of genus Apophallus Lühe, 1909 (Trematoda: Heterophyidae) in Western Pomerania of Poland. Acta Ichthyol. Piscat. 3: $75-89$

WierzBickA, J., GÓRA, D., DubowsKA, G. (1982): Parasites of Acerina cernua (L.) in the Szczecin Firth. Acta Ichthyol. Piscat. 12: $51-61$

WiERzBICKA, J., WierzBICKI, K., PiAsecki, W., ŚmietANA, P. (2005): A comparative study on the parasite fauna of perch, Perca fluviatilis L., collected from a freshwater coastal lake, brackish-water Baltic Sea, and the interconnecting canal. Wiad. parazytol. 51: 295 - 302

WIERZBICKI K. (1970): The parasite fauna of the perch, Perca fluviatilis L. of Lake Dargin. Acta Parasitol. Pol. 18: 45 - 55

WIŚNIEWSKI, W. L. (1958): Characterization of the parasitofauna of an eutrophic lake (Parasitofauna of the biocoenosis of Drużno Lake - part I). Acta Parasitol. Pol. 6: $1-64$

ZANDER, C. D., ReIMER, L. W. (2002): Parasitism at the ecosystem level in the Baltic Sea. Parasitology 124: S119 $-\mathrm{S} 135$ 